\title{
Development and Completeness of Points Of Interest in Free and Proprietary Data Sets: A Florida Case Study
}

\author{
Hartwig HOCHMAIR ${ }^{1}$ and Dennis ZIELSTRA ${ }^{2}$ \\ ${ }^{1}$ University of Florida, Fort Lauderdale/USA·hhhochmair@ufl.edu \\ ${ }^{2}$ University of Florida, Fort Lauderdale/USA
}

This contribution was double-blind reviewed as full paper.

\begin{abstract}
Points of Interest (POI) are used for a variety of analysis tasks in planning, navigation, transportation, tourism, and other areas. For these tasks an understanding of the quality of POI data from different sources is essential. This paper reviews the temporal development and relative completeness of POI from proprietary (TomTom, NAVTEQ, ESRI) and free governmental (TIGER/Line) data sources, as well as that of Volunteered Geographic Information (OpenStreetMap). It highlights the pros and cons for each of these data sets in terms of completeness and currentness. It further analyzes for a subset of features how active the OSM community is in editing and updating previously imported POI from alternative data sources.
\end{abstract}

\section{Introduction}

OpenStreetMap (OSM) ${ }^{1}$ has become a comprehensive source of geodata since its initiation in 2004. OSM is based on volunteered user-generated content and therefore not supervised by an authoritative instance that controls data acquisition. Since there are no obligatory quality standards in OSM for data acquisition and editing, quality assessment of OSM data is necessary to assure their fitness for selected geo-applications. There are ongoing discussions in the OSM community about whether data imports from alternative data sources into OSM are beneficial to the project, or whether this would interfere with data already collected through traditional means, including GPS tracking and tracing from aerial images. The OSM wiki ${ }^{2}$ provides a list of data sets that have been imported into OSM so far, mentioning also the TIGER/Line road data import in 2007, and the import of data from the USGS Geographic Names Information System (GNIS) in the United States. Comparing the temporal development and completeness of OSM features with that from other available data sources will help to determine whether recurring or rather one-time data imports would be beneficial to the project, if at all. This paper will therefore analyze the number of mapped POI features and their temporal development for a variety of free and proprietary data sources. The analysis will be conducted for Florida, since we have access to various publicly available and commercial datasets for this state.

$1 \mathrm{http} / / /$ www.openstreetmap.org/

2 http://wiki.openstreetmap.org/wiki/Import/Catalogue

Jekel, T., Car, A., Strobl, J. \& Griesebner, G. (Eds.) (2013): GI_Forum 2013. Creating the GISociety. (c) Herbert Wichmann Verlag, VDE VERLAG GMBH, Berlin/Offenbach. ISBN 978-3-87907-532-4.

(c) ÖAW Verlag, Wien. eISBN 978-3-7001-7438-7, doi:10.1553/giscience2013s39. 
While there are different aspects of data quality of geographical information, including completeness, logical consistency, positional accuracy, temporal accuracy, and thematic accuracy, the focus of this research is on data completeness. Completeness analyses with regards to OSM data were so far primarily conducted for road network data, either by analyzing data growth within OSM (NEIS et al. 2011, HocHMAIR et al. 2013) or by comparison with alternative data sources (GIRRES \& TOUYA 2010, HAKLAY 2010, KOUKOLETSOS et al. 2012, ZIELSTRA \& HOCHMAIR 2012). The analysis of completeness in OSM POI data is more scarce. STRUNCK (2010) investigated the growth of various OSM POI categories for selected regions in Germany. The comparison with TomTom (formerly TeleAtlas) MultiNet data from 2009 showed that in the analyzed categories OSM had about twice as many POI features as TomTom MultiNet data. Only 1\% of the collected POI in the OSM database had line or polygon geometry, the rest had point geometry. REHRL et al. (2013) assessed for selected regions in Austria the growth of POI, and that of OSM features in general, through detailed analysis of actions performed by individual contributors on the OSM database. MÜLLIGANN et al. (2011) determine the plausibility of new OSM amenity points based on surrounding POI features using a spatial-semantic interaction measure.

POI are used for a multitude of spatial analysis tasks. One of these tasks is to determine a neighborhood's accessibility for different transportation modes, where accessibility describes the ability of people to reach and participate in activities. It is closely related to the number of trip destinations that a traveler can reach within a given time limit. Its analysis has recently been extended to non-motorized traffic, including walking, cycling and public transportation (IACONO et al. 2010), and the generation of walkability ${ }^{3}$ and bikeability ${ }^{4}$ score maps. Closely related to POI are landmarks, which are salient objects in the environment that aid the user in navigating and understanding space. Landmarks are commonly used in human generated route directions and the communication of routes (LOVELACE et al. 1999). Therefore extracting landmarks from POI data and other spatial datasets is of relevance for the automated generation of user friendly route directions (DUCKHAM et al. 2010). Recent approaches classified and identified landmarks from crowd sourced image collections, such as Flickr (LI et al. 2009). RICHTER \& WINTER (2011) developed a prototype editor that allows a user to mark objects in OpenStreetMap as landmarks along with several identifying factors, such as shape and size.

\section{Data Sources: POI Classification and Development}

\subsection{Geographic Names Information System}

The Geographic Names Information System (GNIS) ${ }^{5}$ is the official geographic name repository of the US and the geographic names component of The National Map. It contains records on more than 2 million geographic names in the US and further keeps entries of names and locative attributes of features that no longer exist. The latter are identified by the word "historical" in parentheses after the official name. The current point dataset can be freely downloaded from the GNIS Web site in delimited text file format. The GNIS dataset

\footnotetext{
3 http://www.walkscore.com/

4 http://www.walkscore.com/bike/

5 http://nhd.usgs.gov/gnis.html
} 
from January 2013 contains 54201 features for Florida which are grouped into 51 categories (one of them is labeled "Unknown"). Removing the 185 features without geographic coordinates gives 54016 features (Table 1), from which 551 are labeled historical. Several GNIS feature classes were imported to OSM in 2009, including airports, golf-courses, and hospitals, as described further below.

Table 1: Number of features in GNIS feature classes for Florida

\begin{tabular}{lr|lr|lr|lr}
\hline Category & Count & Category & Count & Category & Count & Category & Count \\
\hline Airport & 956 & Census & 907 & Hospital & 517 & School & 5446 \\
Arch & 2 & Channel & 430 & Island & 1746 & Sea & 2 \\
Area & 6 & Church & 8161 & Lake & 4097 & Spring & 130 \\
Bar & 210 & Civil & 788 & Levee & 32 & Stream & 2978 \\
Basin & 74 & Cliff & 49 & Locale & 4173 & Summit & 151 \\
Bay & 780 & Crossing & 284 & Military & 134 & Swamp & 1001 \\
Beach & 60 & Dam & 15 & Mine & 30 & Tower & 995 \\
Bend & 53 & Falls & 1 & Park & 1815 & Trail & 38 \\
Bridge & 206 & Flat & 97 & Populated Place & 8770 & Unknown & 2 \\
Building & 5277 & Forest & 12 & Post Office & 452 & Valley & 35 \\
Canal & 245 & Gap & 4 & Reserve & 18 & Well & 1 \\
Cape & 745 & Gut & 427 & Reservoir & 135 & Woods & 4 \\
Cemetery & 1263 & Harbor & 241 & Ridge & 21 & & \multicolumn{5}{|c}{ Total } & $\mathbf{5 4 0 1 6}$
\end{tabular}

\subsection{OpenStreetMap}

In OSM coding, elements (nodes, ways, and relations) are described through tags. Each tag consists of a key and a value which are free format textual fields. A key in OSM can be considered a superconcept (e.g. landuse) and the value a subconcept (e.g. cemetery), among other interpretations. While OSM uses a total of 26 primary feature keys including building, highway, or landuse, there is no POI key. Instead, POI must first be identified from a list of commonly used OSM map features ${ }^{6}$, and then be extracted through corresponding keyvalue pairs from the OSM database. We used the list of POI key-value pairs suggested by STRUNCK (2010), which are closely tied to POI available in the TomTom MultiNet dataset, with a few additions. For the analysis, the OSM full history planet dump file was downloaded from planet.openstreetmap.org and processed to extract the necessary information for the years 2008 to 2011 . Additionally, a planet dump file was retrieved and processed for 2012. Next OSM point and polygon features matching the selected key-value pairs were extracted for February 1st for each of the five years. The number of point and polygon features in selected OSM feature classes is listed in Table 2. While there are few features mapped in 2008, the increasing number of mapped features from thereon suggests continuous OSM community mapping activities. The sharp increase in 2010 point data is caused by a GNIS data import during 2009 for several feature classes (underlined values). Some of the POI features that were originally mapped as points were subsequently replaced by more detailed polygon shapes through on-screen digitizing. In OSM, polygons are stored as polylines, some of which are not properly closed. This prevents measuring the area, and

\footnotetext{
6 http://wiki.openstreetmap.org/wiki/Map_Features
} 
in consequence, comparing feature areas to those of other data sources. Therefore we counted the number of polyline features (and not their area) instead.

Table 2: $\quad$ OSM point and area features for selected POI classes

\begin{tabular}{|c|c|c|c|c|c|c|c|c|c|c|}
\hline \multirow[b]{2}{*}{ key-value } & $\stackrel{N}{\mathscr{O}}$ & $\stackrel{N}{\mathscr{G}}$ & $\stackrel{\stackrel{N}{\varrho}}{\theta}$ & $\stackrel{\stackrel{\Xi}{\Xi}}{\underline{\Xi}}$ & $\underset{N}{\stackrel{N}{S}}$ & $\stackrel{\sim}{\mathscr{O}}$ & $\stackrel{n}{\mathscr{O}}$ & $\stackrel{\stackrel{N}{g}}{\theta}$ & $\stackrel{\stackrel{N}{\Theta}}{=}$ & $\underset{N}{\stackrel{N}{S}}$ \\
\hline & \multicolumn{5}{|c|}{ Point Counts } & \multicolumn{5}{|c|}{ Polygon Counts } \\
\hline aeroway-aerodrome & 6 & 11 & 536 & 664 & 661 & 0 & 0 & 3 & 14 & 17 \\
\hline amenity-car rental & 0 & 0 & 3 & 8 & 10 & & & & & \\
\hline amenity-cinema & 0 & 2 & 26 & 39 & 41 & 0 & 0 & 3 & 6 & 10 \\
\hline amenity-college & 0 & 0 & 5 & 9 & 9 & 0 & 4 & 10 & 18 & 33 \\
\hline amenity-doctor & 0 & 0 & 27 & 31 & 40 & 0 & 0 & 0 & 1 & 3 \\
\hline amenity-fuel & 1 & 42 & 106 & 253 & 340 & 0 & 0 & 21 & 39 & 69 \\
\hline amenity-grave yard & 0 & 0 & $\underline{1179}$ & 1170 & 1149 & 0 & 1 & 7 & 14 & 25 \\
\hline amenity-hospital & 0 & 2 & $\underline{460}$ & 460 & 450 & 0 & 1 & 3 & 10 & 38 \\
\hline amen.-kindergarten & 0 & 0 & $\underline{540}$ & 541 & 544 & & & & & \\
\hline amenity-library & 0 & 3 & $\overline{505}$ & 499 & 495 & 1 & 1 & 14 & 18 & 30 \\
\hline amenity-pharmacy & 0 & 8 & $\overline{65}$ & 129 & 155 & 0 & 0 & 0 & 14 & 31 \\
\hline amen.-worship & 0 & 38 & $\underline{8076}$ & 8060 & 7968 & 0 & 0 & 19 & 75 & 107 \\
\hline amenity-police & 0 & 1 & $\overline{710}$ & 709 & 708 & 0 & 1 & 3 & 7 & 8 \\
\hline amenity-post office & 0 & 5 & $\underline{452}$ & 472 & 475 & 1 & 1 & 5 & 7 & 14 \\
\hline amenity-restaurant & 0 & 35 & 333 & 703 & 904 & 0 & 2 & 42 & 65 & 180 \\
\hline amenity-school & 0 & 1 & $\underline{5384}$ & 5231 & 5074 & & & & & \\
\hline amenity-university & 0 & 0 & $\overline{0}$ & 4 & 3 & 0 & 2 & 4 & 7 & 19 \\
\hline leisure-golf course & 0 & 1 & 5 & 24 & 264 & 1 & 3 & 108 & 274 & 479 \\
\hline leisure-park & 0 & 0 & $\underline{1377}$ & 1307 & 1247 & 0 & 37 & 142 & 434 & 688 \\
\hline shop-car & 0 & 0 & $\overline{0}$ & 12 & 17 & 0 & 0 & 0 & 2 & 1 \\
\hline tourism-attraction & 4 & 10 & 54 & 58 & 134 & 0 & 1 & 109 & 129 & 91 \\
\hline tourism-camp site & 0 & 0 & 5 & 14 & 44 & 0 & 0 & 1 & 2 & 4 \\
\hline tourism-hotel & 1 & 15 & 128 & 186 & 204 & 0 & 1 & 29 & 61 & 159 \\
\hline tourism-museum & 0 & 0 & $\underline{146}$ & 159 & 167 & 0 & 1 & 7 & 14 & 25 \\
\hline TOTAL & 12 & 174 & $201 \overline{122}$ & 20742 & 21103 & 3 & 56 & 530 & 1211 & 2031 \\
\hline
\end{tabular}

The number of GNIS points in OSM was 20273 in 2010, 19823 in 2011, and 19635 in 2012, indicating a slight decline since the GNIS data import in 2009. To assess which edits of the OSM community led to this slight decline, and whether this resulted in an improved data quality, we analyzed for three classes the GNIS points in OSM that disappeared from their location between 2010 and 2011 when searching for the same key-value pairs. More specifically, we used aerial basemaps and OSM 2010 and 2011 points to determine whether (a) the GNIS point was converted to a polygon, (b) the GNIS point was removed because there was no corresponding feature on the ground, (c) the GNIS point was shifted, (d) the GNIS point was correctly retagged, or (e) the removal of the GNIS point was incorrect. Table 3 shows that 2231 out of 2354 OSM 2010 points that were imported from GNIS remained unchanged in 2011, whereas 123 were modified. Based on our analysis, all modifications by the OSM community were correct and improved the data quality for aerodromes and hospitals (columns a-c). As opposed to this too many parks were removed according to our interpretation (column e). The position shifts were usually within a few meters and therefore small compared to the size of the mapped object. In a few cases an OSM 2010 feature was modified in more than one way, e.g. by shifting the point geometry 
and converting the feature to a polygon in addition. Correct retagging was observed for four park features (column d), i.e., from park to stadium.

Table 3: Analysis of community based edits on GNIS imported POI in OSM

\begin{tabular}{lllllllll}
\hline $\begin{array}{l}\text { OSM } \\
\text { Feature } \\
\text { class }\end{array}$ & $\begin{array}{l}\text { \# OSM- } \\
\text { GNIS }\end{array}$ & $\begin{array}{l}\text { \# overlap } \\
\text { with OSM- }\end{array}$ & Diff. & $\begin{array}{l}\text { (a) } \\
\text { Poly- } \\
\text { GNIS 2011 }\end{array}$ & $\begin{array}{l}\text { (b) } \\
\text { gon }\end{array}$ & $\begin{array}{l}\text { (c) } \\
\text { feature }\end{array}$ & $\begin{array}{l}\text { (d) } \\
\text { Retag }\end{array}$ & $\begin{array}{l}\text { (e) } \\
\text { Incorrect } \\
\text { removal }\end{array}$ \\
\hline Aerodrome & 529 & 517 & 12 & 5 & 0 & 7 & 0 & 0 \\
Hospital & 456 & 440 & 16 & 3 & 5 & 8 & 0 & 0 \\
Park & 1369 & 1274 & 95 & 27 & 33 & 13 & 4 & 20 \\
\hline Total & $\mathbf{2 3 5 4}$ & $\mathbf{2 2 3 1}$ & $\mathbf{1 2 3}$ & $\mathbf{3 5}$ & $\mathbf{3 5}$ & $\mathbf{2 8}$ & $\mathbf{4}$ & $\mathbf{2 0}$ \\
\hline
\end{tabular}

Fig. 1 gives illustrations for cases (a) and (b) for hospital features in OSM. Both point features were imported from GNIS in 2009 and retained in the 2010 OSM dataset. Both POI were absent in the 2011 point layer for different reasons. The upper hospital POI was replaced by a digitized polyline reflecting the layout of the area in 2011 (case a). As opposed to this, the hospital POI at the bottom was correctly removed and also not replaced by a polygon, since there is no hospital in the area around the point (case b).

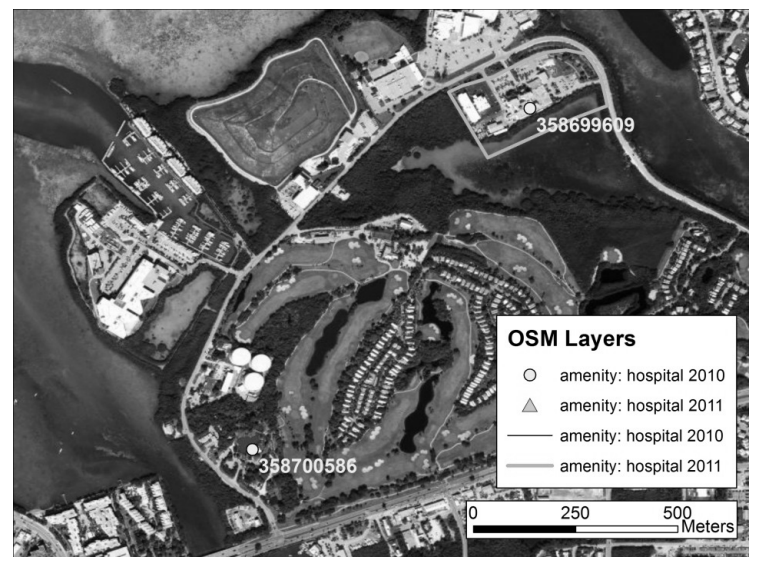

\subsection{TIGER/Line}

TIGER/Line data are freely available for download in shapefile format for the years 20072012 from the US Census Bureau Web site ${ }^{7}$. The TIGER/Line shapefiles contain geographic linear, areal, and point features such as streets, railroads, rivers, lakes, and landmarks (airports, schools, etc.). Landmark features and attributes are available by state and split into area and point landmark shapefiles. The Census Bureau adds landmark features to the database on an as-needed basis and makes no attempt to ensure that all instances of a particular feature are included. Landmarks include airports, cemeteries, locales, populated places, pillars and summits from GNIS. Features in TIGER/Line are classified through a

\footnotetext{
7 http://www.census.gov/geo/maps-data/data/tiger-line.html
} 
5-digit MAF/TIGER Feature Class Code (MTFCC) introduced in 2007. The Florida shapefiles contain 28 point feature classes and 42 polygon feature classes with at least one entry. Visual inspection of the data reveals some inhomogeneity in data completeness. Entries are very scarce in some point (e.g. fire department, landfill, golf course) and area (e.g. hospital, hotel, airport) feature classes, whereas others (those imported from GNIS, such as cemetery) are comprehensive. The number of features recorded in point shapefiles does not vary much over the years except when GNIS data were imported in 2009. Area landmarks showed some increase in 2012 for the feature classes hospital, juvenile institution, prison, and university/college (Table 4).

Table 4: $\quad$ Selected TIGER/Line point and polygon feature classes

\begin{tabular}{|c|c|c|c|c|c|c|c|c|c|c|}
\hline & 芯 & 词 & $\stackrel{\stackrel{\varrho}{g}}{g}$ & 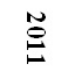 & $\stackrel{\text { 怘 }}{\text { N }}$ & 䓵 & 苛 & $\stackrel{\stackrel{\varrho}{\varrho}}{\varrho}$ & $\stackrel{\widetilde{g}}{\varrho}$ & $\stackrel{N}{\stackrel{N}{N}}$ \\
\hline MTFCC & \multicolumn{5}{|c|}{ Point Counts } & \multicolumn{5}{|c|}{ Polvgon Counts } \\
\hline K1227-Hotel, Motel, $\ldots$ & & & & & & 1 & 1 & 1 & 1 & 1 \\
\hline K1231-Hospital.. & 27 & 27 & 27 & 27 & 28 & 5 & 5 & 5 & 5 & 5 \\
\hline K1237-State Prison, ... & 4 & 4 & 4 & 4 & 4 & 0 & 0 & 0 & 0 & 8 \\
\hline K2193-Fire Department & 0 & 1 & 1 & 1 & 1 & & & & & \\
\hline K2451-Airport/Airfield & 112 & 580 & 575 & 575 & 574 & 114 & 122 & 122 & 122 & 1 \\
\hline K2540-University,... & & & & & & 0 & 1 & 1 & 1 & 9 \\
\hline K2582-Cemetery & 20 & 1098 & 1098 & 1098 & 1095 & 757 & 809 & 809 & 809 & 8 \\
\hline K3544-P1. of Worship & 46 & 46 & 46 & 46 & 46 & 5 & 5 & 5 & 5 & 4 \\
\hline
\end{tabular}

\subsection{TomTom MultiNet}

We analyzed TomTom's MultiNet 2010 ( $3^{\text {rd }}$ quarter $)$ and 2012 ( $3^{\text {rd }}$ quarter $)$ datasets for Florida, which are provided in point shapefile format. POI can be searched by a FEATTYP variable. While the $3^{\text {rd }}$ Quarter of 2010 dataset provides data in 37 POI feature classes, this was reduced to 12 core infrastructure POI categories, such as hospital, airport, ferry terminal, or golf course, after the 2010 releases. All other POI categories that were once part of the MultiNet database would from there on have to be purchased separately in the MultiNet POI enhancement product. The MultiNet 2010 data was missing some categories (e.g., doctor, dentist, car-rental, or zoo) compared to the MultiNet 2009 product analyzed by STRUNCK (2010), possibly due to a change in licensing policy. TomTom datasets are also distributed within ESRI products as described further below.

\subsection{ESRI Data \& Maps and Streetmap North America}

This proprietary ESRI product is the most comprehensive POI data source analyzed and contains two components, i.e. ESRI Data \& Maps USA, and Streetmap North America (NA). Streetmap NA is tailored for routing applications and based on 2007 TomTom streets that were enhanced by ESRI and TomTom. It contains an "institutions" feature class which resembles point shape POI. The feature class is categorized by the older TIGER Feature Class Codes (FCC), has 8 classes, and contains 6662 features for Florida in the 2012 dataset. Similarly, ESRI Data \& Maps USA contains an "institut" feature class that is additionally categorized by TomTom's more refined POI classification scheme for recent years. With a total of 52796 POI for Florida it contains significantly more features than Streetmap NA. ESRI Data \& Maps USA uses several landmark and POI layers from TomTom and 
other sources, such as GNIS or the National Atlas of the US. Both ESRI products also contain other POI feature classes, such as a transportation terminal point feature class or a landmarks polygon feature class. Further, additional feature classes are available with the Data \& Maps USA product, including golf, church, cemetery, or schools, which provide more detail than the "institut" feature class alone. Table 5 compares the number of point features found in selected feature classes of Streetmap NA and ESRI Data \& Maps USA in different years, with data sources being indicated in the footnote. The horizontal grouping follows the FCC scheme used in the "institutions" and "institut" feature classes. Feature counts reported come from these two feature classes, except where alternative feature classes (e.g. from GNIS) resulted in larger feature counts. Table 5 reveals that Streetmap NA 2012 and Data \& Maps 2008 follow the same FCC classification scheme with no subclasses. However, Data \& Maps provides a more refined classification in recent years, especially for educational institutions, with a significant increase in total feature numbers. The total at the bottom counts also features from classes not shown here, such as bus terminals.

Table 5: Point features from ESRI Streetmap North America and ESRI Data \& Maps USA

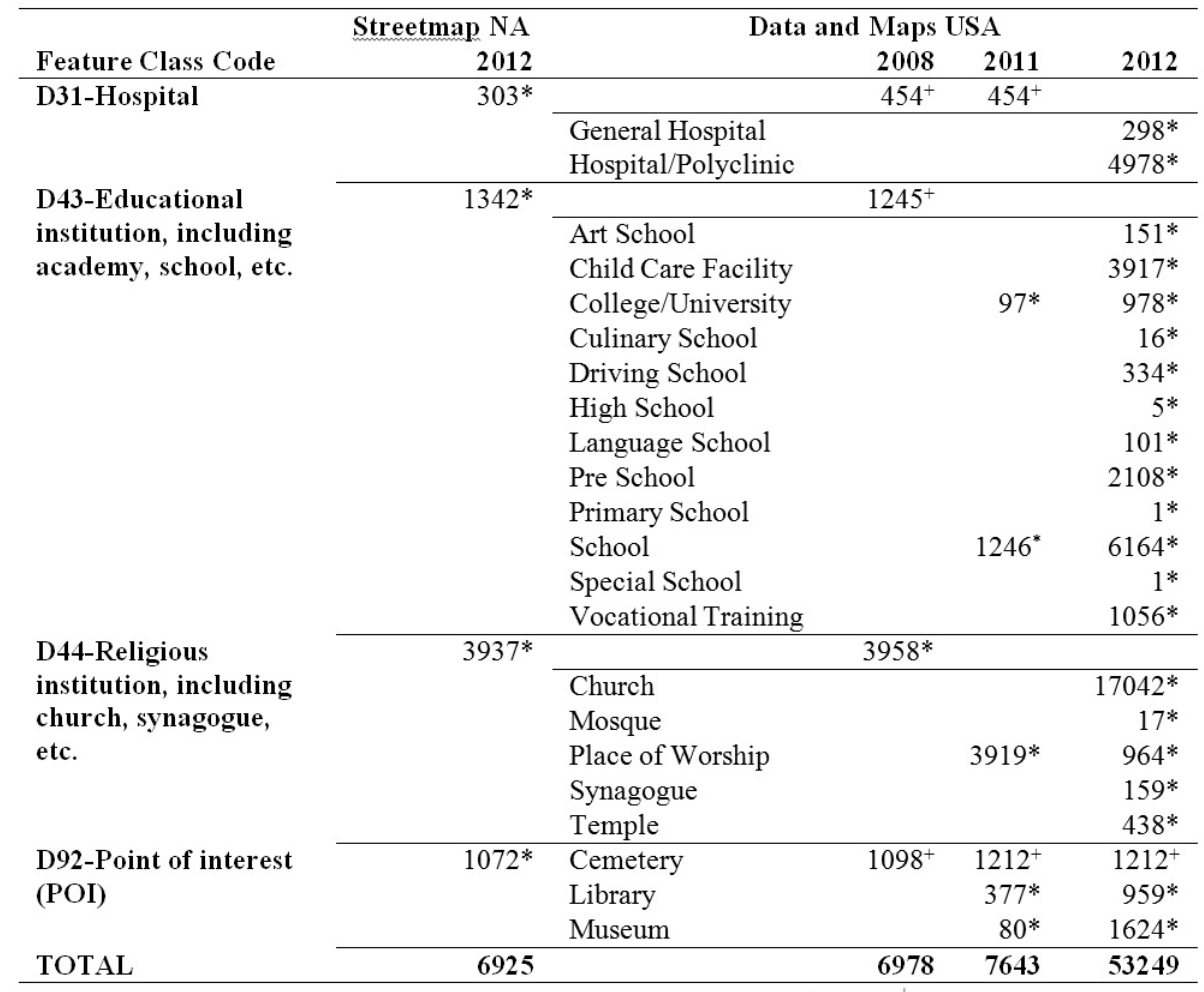

Data sources: * TomTom (provided through "institutions" or "institut" feature class), ${ }^{+}$GNIS

\subsection{NAVTEQ NAVSTREETS}

NAVTEQ provides 12 POI related point feature classes as part of the NAVSTREETS product. The product contains also a Building/Landmark Features polygon file, which is only 
sparsely populated and missing further subcategories. It was therefore not analyzed in more detail. The 12 point feature classes cover 56 facility types and provide the most detailed classification scheme among all analyzed point data sources except for OSM. The total number of 119310 POI features for Florida ( $2^{\text {nd }}$ quarter of 2012) is the highest among all data sources. We had access to NAVTEQ POI files from 2010 ( $2^{\text {nd }}$ quarter $), 2011\left(1^{\text {st }}\right.$ quarter), and 2012 ( $2^{\text {nd }}$ quarter). Comparison between different years shows a consistent grouping of POI facility types into feature classes. Feature counts change slightly between analyzed quarters, suggesting a continuous updating process of the data. Individual POI facility types, such as library, can be accessed through NAVTEQ facility codes. In some instances a feature was mapped twice at the same location through two POIs associated with different facility types, e.g. facility codes 5000 (Business facility) and 6000 (Bank).

\section{Comparison of Different Data Sources}

This section compares the number of point features in selected classes between different data sources. The focus is on point features which are available for all data sources. Table 6 refers to 15 selected OSM POI classes through their key-value pairs. It shows the number of feature counts for OSM and corresponding classes in other data sources for 2012.

Table 6: Point feature count comparison between different data sources for 2012

\begin{tabular}{|c|c|c|c|c|c|}
\hline OSM key-value & OSM & $\begin{array}{l}\text { TIGER/ } \\
\text { Line }\end{array}$ & $\begin{array}{l}\text { TomTom } \\
\text { MultiNet }^{1}\end{array}$ & $\begin{array}{l}\text { NAVTEQ } \\
\text { NavStreets }\end{array}$ & $\begin{array}{l}\text { ESRI Maps } \\
\text { \& Data }\end{array}$ \\
\hline aeroway-aerodrome & 661 & 574 & 72 ศ & 305 & $34^{\mathrm{a}}$ \\
\hline amenity-fuel & 340 & & & 5980 & \\
\hline amenity-grave yard & 1149 & 1095 & 1064 & & $1212^{+}$ \\
\hline amenity-hospital & 450 & 28 & $304^{\leftarrow}$ & 417 & $5276^{*}$ \\
\hline amenity-library & 495 & & $378^{\circ}$ & 490 & $959 *$ \\
\hline amenity-pharmacy & 155 & & & 4169 & \\
\hline amenity-place of worship & 7968 & 46 & $3921^{\top}$ & & $18620 *$ \\
\hline amenity-police & 708 & 1 & $1^{\uparrow}$ & 334 & \\
\hline amenity-school & 5074 & 94 & $1246^{\circ}$ & 4772 & $6164 *$ \\
\hline amenity-theatre & 64 & & & & \\
\hline leisure-golf course & 264 & 20 & $1083^{\star}$ & 1124 & $403^{+}$ \\
\hline leisure-park & 1247 & 46 & & & \\
\hline shop-car repair & 25 & & 28 & 12950 & \\
\hline tourism-museum & 167 & & $81^{\bullet}$ & & $1624^{+}$ \\
\hline railway-station & 142 & & 252 & 87 & $204^{*}$ \\
\hline
\end{tabular}

${ }^{1}$ Data sources: ${ }^{\mathfrak{9}}$ TomTom MultiNet 2010; default year $=2012$

${ }^{2}$ Data sources: ${ }^{\text {a }}$ National Atlas, ${ }^{*}$ TomTom, ${ }^{+}$GNIS,

The table is an attempt to assess the relative completeness between data sources. One challenge was to match the different classification schemes for feature counts. For example, while the class "place of worship" in OSM, TIGER, and TomTom MultiNet contains amenities for all religions (e.g. church, mosque, temple, etc.), ESRI Maps \& Data 2012 provides a more detailed classification (see Table 5). These detailed classes in ESRI Maps \& Data were therefore aggregated to match place-of-worship counts in other data sources. In some cases classes cannot be aggregated, since data are not available. For example, in the case of aerodrome (airport), GNIS, which has been imported into the OSM aerodrome class, 
includes also unpaved airfields. So does the TIGER K2451-Airport or Airfield class. As opposed to this, the National Atlas contains fewer features, i.e. international, regional, and some military airports. Similarly, TomTom MultiNet data contains primarily international, regional, and municipal airports (with paved runways), but no records on local airfields. Another example is the class hospital, where some providers also include polyclinics in this category in addition to general hospitals. Blanks in Table 6 indicate cases where classes corresponding to OSM are not available in other data sources.

Some patterns of relative completeness can be identified from Table 6. OSM, since it is an open platform, covers the largest variety of feature classes. It performs particularly well in feature classes with GNIS data imports. ESRI Maps \& Data have the highest feature count when using GNIS or TomTom data, except for golf course and railway stations. Though OSM and ESRI rely partially on GNIS data, their feature counts in corresponding classes do not exactly match, probably due to further data edits in both sources. TomTom data, when included in different products (MultiNet vs. ESRI Maps \& Data) provide a different level of detail. In this sample, the TomTom based feature classes in the ESRI product contain generally more details than in the MultiNet data set. NAVTEQ provides the most comprehensive list of POI feature types among the commercial data providers, yet maintaining a high level of detail, especially in business related categories (e.g., gas station, pharmacy, car repair shops). TIGER/Line shows the fewest features in most classes. Further it reveals only few changes over the years, indicating that feature classes are not continuously updated. These two facts limit TIGER POI for use in spatial analysis tasks.

\section{Summary and Outlook}

A large variety of POI data providers makes it a challenge to identify the most appropriate data set for a given analysis task. This study reviewed and characterized some commonly used POI data sets in the US. It could be observed that data classification schemes in products of the different proprietary providers change over time, which makes a reconsideration of data products necessary from time to time. The analysis shows that no single data source outperforms another in all aspects, and that therefore the choice of the best dataset will depend on the given task (besides the monetary aspect associated with proprietary data). Further, POI features may have to be complemented with other sources, such as census or job data for GIS analysis. The presented research provides a first assessment of OSM POI completeness in Florida. Since OSM, TIGER and GNIS are freely available for whole US, this would allow us to expand this analysis to a larger geographic area in the future. Temporal analysis of OSM data for Florida between 2008 and 2012 showed a continuous data growth in most feature classes. Even with POI data that are imported from GNIS data, the OSM community is actively working on improving their data quality through further data edits. Therefore in the context of POI it seems that one-time data imports from alternative data sources are sufficient, as long as this import provides a satisfactory level of detail and other OSM data are not yet present. For future work we plan to expand this preliminary analysis to other parts of the US, and to identify the effect of population density on relative data completeness. 


\section{References}

DuCKHAM, M., WinTER, S. \& RoBInSON, M. (2010), Including landmarks in routing instructions. Journal of Location Based Services, 4 (1), 28-52.

GirRes, J.-F. \& TOUYA, G. (2010), Quality Assessment of the French OpenStreetMap Dataset. Transactions in GIS, 14 (4), 435-459.

HAKLAY, M. (2010), How good is Volunteered Geographical Information? A comparative study of OpenStreetMap and Ordnance Survey datasets. Environment and Planning B, Planning and Design, 37 (4), 682-703.

Hochmair, H. H., Zielstra, D. \& NeIs, P. (2013), Assessing the Completeness of Bicycle Trails and Designated Lane Features in OpenStreetMap for the United States and Europe. Proceedings of the Transportation Research Board - 92nd Annual Meeting, Washington, D.C., January 13-17, 2013, Transportation Research Board of the National Academies.

IACONO, M., KRIZEK, K. J. \& EL-GENEIDY, A. (2010), Measuring non-motorized accessibility: issues, alternatives, and execution. Journal of Transport Geography, 18 (1), 133140.

Koukoletsos, T., Haklay, M. \& Ellul, C. (2012), Assessing Data Completeness of VGI through an Automated Matching Procedure for Linear Data. Transactions in GIS, 16 (4), 477-498.

Li, Y., Crandall, D. J. \& Huttenlocher, D. P. (2009), Landmark Classification in Large-scale Image Collections. Proceedings of IEEE 12th International Conference on Computer Vision (ICCV), Kyoto, Japan, ACM.

Lovelace, K. L., Hegarty, M. \& Montello, D. R. (1999), Elements of Good Route Directions in Familiar and Unfamiliar Environments. In FrEKSA, C. \& MARK, D. M. (Eds.), Conference on Spatial Information Theory (COSIT'99) (LNCS 1661). Berlin, Springer, 65-82.

Mülligann, C., Janowicz, K., Ye, M. \& LeE, W.-C. (2011), Analyzing the SpatialSemantic Interaction of Points of Interest in Volunteered Geographic Information. In: Egenhofer, M. J., Giudice, N. A., Moratz, R. \& Worboys, M. F. (Eds.), Conference on Spatial Information Theory (COSIT 2011) (LNCS 6899). Berlin, Springer, 350-370.

NeIS, P., ZIELSTRA, D. \& ZIPF, A. (2011), The Street Network Evolution of Crowdsourced Maps: OpenStreetMap in Germany 2007-2011. Future Internet, 4 (1), 1-21.

Rehrl, K., Gröchenig, S., Hochmair, H. H., Leitinger, S., Steinmann, R. \& Wagner, A. (2013), A Conceptual Model for Analyzing Contribution Patterns in the Context of VGI. In: KRISP, J. M. (Ed.), Progress in Location Based Services (Lecture Notes in Geoinformation and Cartography). Springer, Berlin, 373-388.

RichTER, K.-F. \& WINTER, S. (2011), Harvesting User-Generated Content for Semantic Spatial Information: The Case of Landmarks in OpenStreetMap. Proceedings of Surveying \& Spatial Sciences Biennal Conference 2011, Wellington, New Zealand.

STRUNCK, A. (2010), Raumzeitliche Qualitätsuntersuchung von OpenStreetMap (MSc Thesis, Geographisches Institut, Rheinische Friedrich-Wilhelms-Universität Bonn, Bonn, Germany).

Zielstra, D. \& Hochmair, H. H. (2012), Using Free and Proprietary Data to Compare Shortest-Path Lengths for Effective Pedestrian Routing in Street Networks. Transportation Research Record: Journal of the Transportation Research Board, 2299, 41-47. 